\title{
Material Processing with Femtosecond Laser Pulses for Medical Ap- plications
}

\author{
S. Fiedler ${ }^{1}$, R. Irsig $^{1}$, M. Gieseke ${ }^{2}$, M. Vehse ${ }^{3}$, V. Senz ${ }^{4}$, A. W. Oniszczuk ${ }^{1}$, J. Tiggesbäumker ${ }^{1}$, C. Schuster ${ }^{1}$, A. V. \\ Svanidze $^{1}$, N. Rothe ${ }^{1}$, S. Kaierle ${ }^{2}$, M. Hustedt ${ }^{2}$, H. Haferkamp ${ }^{2}$, K. Sternberg ${ }^{4}$, K.-P.Schmitz ${ }^{4}$, H. Seitz ${ }^{3}$, S. Lochbrun- \\ ner $^{1}$, K.-H. Meiwes-Broer ${ }^{1}$ \\ ${ }^{1}$ Institute of Physics, University of Rostock, 18051 Rostock, Germany, meiwes@uni-rostock.de \\ ${ }^{2}$ Materials and Processes Department, Laser Zentrum Hannover e.V., 30419 Hannover, Germany \\ ${ }^{3}$ Chair of Fluid Technology and Microfluidics, University of Rostock, 18059 Rostock, Germany \\ ${ }^{4}$ Institute for Biomedical Engineering, University of Rostock, 18119 Rostock, Germany
}

\begin{abstract}
Medical implants require functional surfaces in order to ensure biological compatibility. Micrometer-sized surface structures are needed in particular for a successful adaptation on a cellular basis. Pulsed laser light sources with sub-ps pulse durations are promising tools exceeding the precision in material processing of nowadays mechanical methods. Our three-axis-scanner allows arbitrary focus positioning on the sample for material processing. Intense ultrashort near infrared laser pulses with adjustable pulse envelopes and variable focusing conditions are used for the material ablation. Moreover, process parameters like pulse energy, pulse repetition rate and focusing conditions are adjusted to adapt the laser parameters to the specific requirements regarding geometry and materials in use. Prototype drug depots have been machined by laser surface structuring.
\end{abstract}

\section{Introduction}

Material modifications on the $\mu \mathrm{m}$-scale are required in many applications, e. g. in micromechanics or modern medical implant technologies. Pulsed laser light sources are powerful tools dedicated to replace conventional methods of mechanical processing in many cases $[1,2]$.

In laser machining the working area is confined to the laser focus with minimal effects on the functionality of the surrounding surface. Even higher precision becomes available for ultrashort femtosecond (fs) laser pulses by a reduction of heat transfer into the material due to the short interaction time [3]. Furthermore the high intensity of femtosecond laser pulses leads to a different ablation mechanism that in principle allows for processing arbitrary materials. In order to achieve this it is necessary to adapt the laser parameters for each sample. Especially when applying shaped laser pulses an improved precision is accessible [4]. The goal of this project is to optimize the working conditions and the quality of the machining process with these ultrashort pulsed lasers. In particular, biocompatible materials were chosen for the creation of hole formations in micro-sized needles and plates [5].

\section{Methods}

A commercial laser system with a center wavelength of $793 \mathrm{~nm}$ was used to supply ultrashort laser pulses for the material processing. Samples are put into a holding system which can freely move along 3 different axes so that a 3 dimensional structuring of the material is possible. The experiments were carried out in ambient air (Image 1).

As a prototype drug depot for medical applications we machined micro needles produced by Selective Laser Micro
Melting (SL $\mu \mathrm{M})$ as described in Ref. [6]. The micro needles were fabricated out of biocompatible stainless steel alloy $316 \mathrm{~L}$ (1.4404) supplied by a $316 \mathrm{~L}$ substrate plate. The needles featured a diameter between $80 \mu \mathrm{m}$ and $320 \mu \mathrm{m}$ and a height of about $1 \mathrm{~mm}$. Due to their finite wall thickness, the needles with smaller diameters were fully dense without any cavities. For a use as drug depots they needed to be opened up from the top.

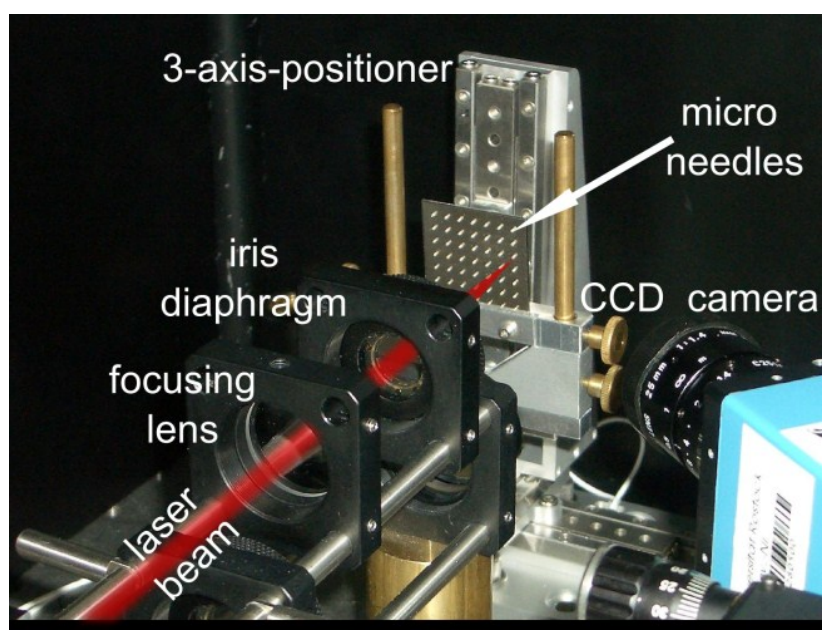

Image 1 Laser machining setup for experiment on micro needles

For the generation of larger holes different procedures can be applied. One possibility is to make a 2-dimensional scan where the spacing between several single holes or deepenings arranged in a circle is reduced until finally one larger hole is formed [7]. However this will normally result in a reduced quality of the generated structure and / or would require much effort to optimize the technique for 
the material ablation process. A second idea is just to change the focusing conditions by the use of different lens optics in order to change the radius within which the ablation takes place. In addition to the simplified working conditions this second method results in a much faster process speed if the laser intensity can be adjusted accordingly.

For the first experiments on the micro needles as also described in Ref. [6] the possibilities were quite limited. In another approach we produced drug depots with up to $100 \mu \mathrm{m}$ in diameter on a bare 316L stainless steel sample surface. These drug depots were subsequently loaded with acetylsalicylic acid (ASS) by the use of a drop-on-demand printhead (see Ref. [5]).

Two precision rotation mounts were implemented to align the micro needles parallel to the incoming laser beam. The position of the laser spot that is focused onto the sample was controlled by a magnifying CCD camera. The sample position could be controlled by software with a resolution of $1 \mu \mathrm{m}$ (Image 1). For machining laser pulses of 150$200 \mathrm{fs}$ pulse width and an energy of $150 \mu \mathrm{J}$ per pulse were used. With a repetition rate of $1 \mathrm{kHz}$ the pulses were guided onto the sample using varying focusing conditions.

\section{Results}

The drug depots could be precisely placed onto the front edge of the micro needles. One of the machining results is shown on Image 2, where a drug depot with a diameter of approximately $50 \mu \mathrm{m}$ is drilled from the top of the needle.

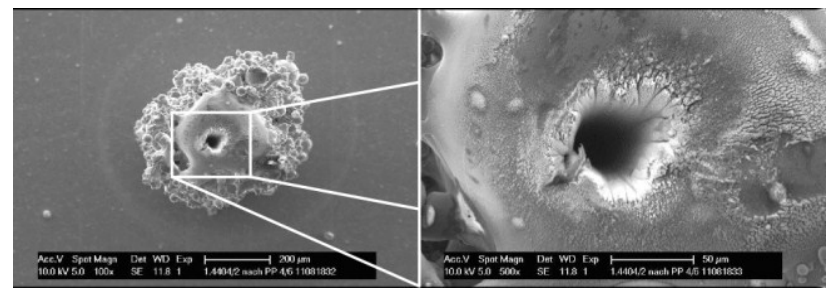

Image 2 Drug depot on micro needle

In order to supply a distinct container volume for solved drugs, specific dimensions have to be realized. A laser parameter study has been carried out (Image 3) to find out the right conditions for the drug depots which were used for the loading process with ASS presented in Ref. [5]. The shown dependencies of the hole depth on the number of pulses and on the pulse intensity were measured for different focusing conditions. The same was done for the hole diameters.

Drug depots machined with the femtosecond laser revealed steeper outer walls as compared to the previously fabricated depots by the use of a nanosecond laser system. This leads to a more cylindrically type of shape. Likewise the precision of the machining process increases with the application of femtosecond laser ablation.

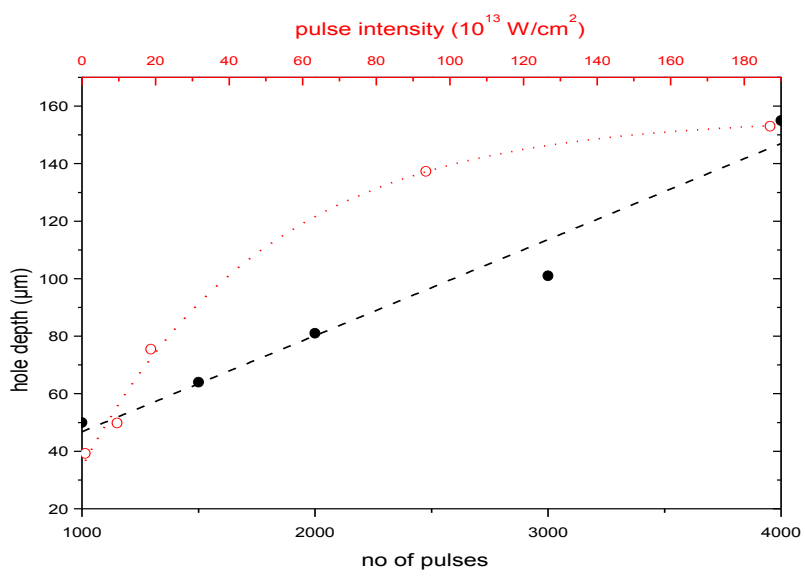

Image 3 Hole depth versus pulse intensity (red curve) and the number of pulses (black curve) respectively for stainless steel 316L. Pulse length: $130 \mathrm{fs,} \mathrm{focal} \mathrm{distance:}$ $100 \mathrm{~mm}$.

\section{Conclusion}

High quality material processing results with structure sizes near the diffraction limit are achieved with ultrashort laser pulses. According to the various application potential we successfully conduct experiments on medically relevant representatives of different material classes like $316 \mathrm{~L}$ stainless steel and silicone. With knowledge of the suitable ablation conditions micro-sized needles (Image 2) and drug depots on stainless steel plates [5] are processed as prototypes for a specific medical application. Material processing with femtosecond laser pulses provides versatile opportunities in the fabrication of medical implants.

\section{Acknowledgements}

The Federal Ministry of Education and Research (BMBF) of the Federal Republic of Germany is gratefully acknowledged for the financial support. REMEDIS is part of the "Leading-Edge Research and Innovation in the New German Länder" programme (Project number FKZ: 03IS2081) with the goal of a "Higher quality of life through novel micro-implants".

\section{SPITZENFORSCHUNG\&INNOVATION

I N DEN NEUEN L A N D E R N

\section{References}

[1] Krüger, J. and Kautek, W.: The femtosecond pulse laser: a new tool for micromachining, Laser Physics 9, 30 (1999)

[2] Irsig, R. et al: Oberflächenstrukturierung mit ultrakurzen Laserpulsen, BiomedTech 55, S1, 46-47 (2010)

[3] Chichkov, B. N. et al., Appl. Phys. A 63, 109 (1996)

[4] Englert, L. et al., Appl. Phys. A 92, 749 (2008) 
[5] Vehse, M. et al., Loading method for discrete drug depots on implant surfaces, Jena: BMT 2012 (2012)

[6] Gieseke, M. et al.: Additive Manufacturing of Drug Delivery Systems, Jena: BMT 2012 (2012)

[7] Dausinger, F. et al., Micro-machining with ultrashort laser pulses: From basic understanding to technical applications, Proceedings of the SPIE 5147, 106 (2003)

[8] Svanidze, A. et al.: Material processing with shaped femtosecond laser pulses, Jena: BMT 2012 (2012) 